\title{
MERITOCRACY, EFFICIENCY, INCENTIVES AND VOTING IN COOPERATIVE PRODUCTION: A SURVEY*
}

\author{
Carmen Beviá \\ Luis C. Corchón \\ Universidad de Alicante \\ Universidad Carlos III de Madrid
}

September 17, 2017.

\begin{abstract}
This paper surveys selectively several contributions to the understanding of how cooperatives may cope with the interplay between meritocracy and efficiency when public decisions are taking by voting and the supply of labor is freely decided by each member. This outlines the main tradeoff faced by cooperatives. In particular, the degree of meritocracy is limited by three factors. 1) Efficiency because too much meritocracy encourages too much work from the socially optimal point of view). 2) Meritocracy encourages sabotage. 3) Voting because workers may prefer inefficient reward schemes as long as they are individually profitable.
\end{abstract}

Keywords: Cooperative Production, Growth, Meritocracy, Efficiency, Incentives,Voting.

JEL classification: D2, D9, O4.

Corresponding author: Carmen Beviá. e-mail: Carmen.bevia@gmail.com

Telephone: +34965903614

\footnotetext{
${ }^{*}$ This paper is dedicated to Joaquim Silvestre from whom we lerned so much, specially in this topic. The first author acknowledges financial support from ECO2014 53051, SGR2014-515. The second author acknowledges financial support from ECO2014-57442-P, and financial support from the Ministerio Economía y Competitividad (Spain), grants S2015/HUM-3444 and MDM 2014-0431.
} 


\section{Introduction}

Mainstream economic theory assumes that firms are own by shareholders who are interested in maximizing profits. ${ }^{1}$ This covers a fair amount of ground but it leaves outside cooperative production in which a group of people (say, workers) owns a production facility (a technology, a plot of land) and a certain input (labor) which has alternative uses (another facility, leisure,...). Inputs and technology produce output. The reader may think of a cooperative owning a flat and using labour to produce teaching services. ${ }^{2}$

The theory of cooperative production, that we will survey selectively in this paper, assumes that the owners of the production facility satisfy all the customary assumptions of neoclassical economics: they have preferences over consumption and leisure that are representable by a (differentiable, increasing and concave) utility function and, when time comes, they maximize as relentlessly as they do their peers in mainstream models. Technology will be represented by a production function and the cooperative cannot influence the price of the good they sell. Thus, it will come to no surprise that many of the problems associated with capitalism resurface in this framework: Sabotage, static and dynamic inefficiencies, wrong incentives, even unemployment and exploitation! So what is new here?

What is new is twofold. On the one hand, we have to determine how surplus is distributed. In the standard model, profits are distributed according to exogenously given proportions. On the other hand, having removed the assumption of profit maximizing firms, we have to describe a criterion to choose among production plans. Let us consider these two issues in turn.

Distributional issues are dealt with the concept of a Sharing Rule (Sen (1966), Moulin (1987), Roemer and Silvestre (1993)). This is a function mapping effort, labor or any contribution made by each worker into the share of the output of each worker. For instance we may give every worker the same share, irrespective of their contributions. This is the Egalitarian sharing rule. Or we may equal this share to the share of her effort with respect to total effort. This is the Proportional sharing rule. Or we might combine both. As we will see, there is a host of sharing rules and, unless additional reasons are supplied, there is no particular reason to prefer one to another. This

\footnotetext{
${ }^{1}$ Despite the fact that profit maximization agrees exactly with shareholders interests only in special cases, like large economies, see Hart (1979).

${ }^{2}$ In fact the work of Roemer and Silvestre on this topic was motivated by their membership to a wine cooperative in Davis.
} 
implies two things: One, that theorems which hold for general sharing rules are preferable to those in which a particular form is postulated. Two, that when using a particular rule, we must supply reasons why this rule is chosen. As we will see, efficiency and majority voting are two of such requirements. Axiomatics is another. Kang (1988) showed that for more than two workers, the convex combination of the egalitarian and the proportional sharing rules is the unique differentiable sharing rule which is symmetric and homogeneous of degree zero (i.e. independent of the units in which the input is measured). ${ }^{3}$ Some of the results surveyed in this paper will use this functional form.

The first papers on cooperative production assumed that wage maximization was a reasonable goal, see Ward (1958) and Vanek (1970). But this goal was too extreme in the sense that gave no value to leisure, only consumption. ${ }^{4}$ A more reasonable goal would be that, given a sharing rule, the production plan should be Pareto Efficient inside the coop (Roemer and Silvestre (1993)). Next issue is the non cooperative implementation of such solutions. Sen (1966) proposed a Nash equilibrium in which each worker is free of supplying whatever amount of labor she wants, i.e. a Contribution Mechanism. He proved that equilibrium and efficiency are simultaneously attained by setting the weight of the proportional sharing rule equal to the elasticity of output with respect to labor. The intuition is that, an egalitarian sharing rule gives incentives to work very little. But meritocracy gives incentives to work more than it is socially efficient because an increase in work, not only means more output, it also means that the share of the labour of the person making an extra effort increases. Unfortunately this result only holds when all workers are identical (Beviá and Corchón (2009) and Moulin (2010)). When they are not, the production function must be polynomial of degree at most $n-1$ where $n$ is the number of workers in the cooperative. We present a simplified version of this result. The sharing rule compatible with efficiency is the Incremental Sharing Rule. ${ }^{5}$ Recall that a result, due to Weierestrass, states that every continuous function defined on a closed interval $[a, b]$ can be uniformly approximated as closely as desired by a polynomial function. Thus, when $n$ is large and the production function is continuous, a polynomial production function is

\footnotetext{
${ }^{3}$ Moulin (1987) provided an alternative axiomatization of this sharing rule.

${ }^{4}$ This goal also produced weird comparative static results like an increase in demand decreases total output, see Ward (1958) for details.

${ }^{5}$ This rule has two parts. One part awards each agent with her marginal product as in the Vickrey-Clark-Groves mechanisms. The other part depend on the contributions of other agents. These terms are chosen such that when the production function is polynomial, the incremental sharing rule delivers as much consumption as output.
} 
indeed a good approximation. When it is not, implementation theory (see e.g. Corchón (2017) for a survey) is called to the rescue! Recall that implementation provides tools to analyze decentralized solutions. ${ }^{6}$ In fact Corchón and Puy (2002) showed that for any sharing rule, there is a mechanism whose Nash equilibria yield allocations in the sharing rule that are Pareto efficient for a large set of preferences. $^{7}$ This result appears to imply that, provided that the right mechanism is used, there is no conflict between efficiency and distribution. In other word, even if the sharing rule does not encourage work, a mechanism can be designed to overcome the rational laziness of agents.

However implementation theory assumes that all actions are controlled by the mechanism. When this strong assumption is lifted, sabotage raise its ugly face. As far as we know Nalebuff and Stiglitz (1983) were the first to acknowledge that "In the competitive system.... there are... rewards from engaging in destructive activity" (id. p. 40). In the case of a capitalistic firm, Lazear (1989) pointed out that large differences in salaries encouraged sabotage. Beviá and Corchón (2006) study sabotage under cooperative production and showed that when the possibilities of destruction by sabotage are small in relation to the number of agents, all sharing rules satisfying two mild conditions yield a Nash equilibrium with no sabotage. However, when the possibilities of destruction are not small, the degree of meritocracy that is compatible with the absence of sabotage depends negatively on the degrees of congestion and substitutability among the inputs provided by workers. In any case, the egalitarian sharing rule is safe from sabotage ${ }^{8}$

Having studied how allocative efficiency and sabotage might be used to shrink the set of reasonable sharing rules we now turn our attention to voting. We are motivated by the fact that many decisions in real cooperatives are taken by voting, sometimes delegating the decision in a democratically chosen Board of Directors (BOD). In this case we assume that there is a function mapping any possible composition of the BOD into the set of sharing rules. ${ }^{9}$ Assuming quasi-linear utilities, Corchón and Puy (1998) showed that, to guarantee individual rationality, the weight given to egalitarian sharing rule must be zero. They also showed that under additional conditions, utility

\footnotetext{
${ }^{6}$ Implementation theory looks for means of transmitting the information in the hands of agents such that the game induced among the agents yields the desired outcome when agents behave strategically.

${ }^{7}$ They also show that under "classical" conditions these allocations exist.

${ }^{8}$ This might help to explain why cooperatives tend to offer egalitarian incentive schemes (Kremer [1997]).

${ }^{9}$ This procedure assumes that the decisions of the BOD can be modeled in a convenient black box without inquiring about the methods used by the BOD to achieve decisions. See Reynolds (2000) for a discussion of some of these methods, which include voting.
} 
is single peaked in the weight of the proportional sharing rule. Thus, the peak of the median voter determines the sharing rule. It turns out that in this case, this peak is located in the extreme (i.e. either the proportional rule gets zero weight or the whole weight). ${ }^{10}$ Later on, TorregrosaMontaner (2017) explored the case when a continuum of voters have Cobb-Douglas preferences and agents differ in their wealth endowments. He finds that when wealth distribution exhibits positive (negative) skew, the degree of meritocracy chosen by individuals is lower (higher) than that of the optimum, and coincides with it when the distribution is symmetric. Moreover, efficiency is also reached for extreme levels of returns to scale.

Barberá, Beviá and Ponsati (2015) presented a model of coalition formation where workers choose their reward schemes by majority. This create instability in the organizations in some cases. They also show that, when stable organizational structures exist, coalitions with proportional or egalitarian sharing rules may coexist and the coalitions that are formed are not necessarily segregated.

The literature considered so far, focus in a static cooperative where entry and exit of new and old members and the capital stock are exogenously given. We take these two issues in turn.

Corchón (2005) presented a model of entry and exit in an overlapping generations model in which agents with High $(\mathrm{H})$ and Low $(\mathrm{L})$ productivity are free to enter into the cooperative and live for two periods. In the first period, agents perform a task for which all agents are equally capable. At the beginning of the second period, one of them is (randomly) chosen to be the boss. Other agents might leave the organization if they wish. If the boss is $\mathrm{H}$, she has a positive externality on all other agents. When this externality is large, in a dynamic equilibrium, no matter how likely is that the boss will be $\mathrm{H}$, both classes of workers coexist. The explanation of this fact is that when the externality is large, $\mathrm{L}$ workers receive a large externality from a $\mathrm{H}$ boss so they have incentives to enter. This is the Umbrella Effect. ${ }^{11}$ He also shows that the quality of the members of the organization may change cyclically. Beviá, Corchón and Romero-Medina (2016) introduce Medium productivity workers (M) and, instead free entry, incumbent members can choose

\footnotetext{
${ }^{10}$ In this case the proportional rule is mixed with the Equal Benefit Rule defined below !!!!!

${ }^{11}$ This effect provides an explanation to the casual observation that some intelligent leaders are surrounded by mediocre people. The usual explanation of this fact is that, intelligent people do not like to be surrounded by potential challengers. Without denying that this may be the case in some instances, the umbrella effect provides an alternative explanation: Intelligent bosses provide an umbrella under which the mediocre prosper.
} 
by plurality voting whom to hire and how to share output. They show that in some cases, L agents may yield political power (i.e. majority) to $M$ agents even if they value the future highly. This is what they call the Relinquish Effect. They also show that $\mathrm{H}$ agents may receive less than their individual output, i.e. they are exploited and they may be not hired because their entrance may threaten the dominance of the dominant class. They call this Political Unemployment.

Lastly we consider accumulation of human capital (e.g. schooling). The investment in human capital has two effects. A direct effect for the agents investing and an indirect effect for their descendants. Beviá and Corchón (2017) assume that agents are identical and produce their own capital using the inherited capital and labor. Output is produced by capital alone and is distributed according to a weighted average of the proportional and the egalitarian sharing rules. Suppose first that agents do not care about future generations. Under Cobb-Douglas preferences (i.e. when the elasticity of substitution equals to one) capital grows at a constant rate. This rate depends positively on the taste of consumption and the degree of meritocracy because both encourage effort which in turn produces more capital. For any other value of the elasticity of substitution, there is a steady state value of capital. ${ }^{12}$ When they consider a set up in which workers maximize a discounted sum of utilities they have to assume that preferences are Cobb-Douglas. They show that when future generations do not count much, there is a solution to the intertemporal maximization problem in which the growth rate is constant and larger than the corresponding rate in the zero discounting case. This is due to the fact that when future counts, there are more incentives to invest in capital which in turn stimulates growth. In both cases, meritocracy encourages work which produces human capital, which makes the economy grow. But this does not imply that quick growth is good for social welfare because, possibly, this is achieved at cost of very hard labor. Surprisingly, they prove that Sen's result (1966) resurfaces here: dynamic efficiency is achieved when the weight of the proportional sharing rule equals the elasticity of output with respect to capital.

A running topic throughout this paper is the compatibility of meritocracy, efficiency, incentives to work and voting on income allocation inside the cooperative. While efficiency, incentives and voting are easily identified, the definition of meritocracy is not obvious. Standard definitions of meritocracy stress ability or talent. We think otherwise. Merit should be tied to actions not to

\footnotetext{
${ }^{12}$ When the elasticity of substitution is smaller than one (i.e. consumption and leisure are complements), the steady state is stable, i.e. growth eventually disappears. But if the elasticity of substitution is larger than one (i.e. consumption and leisure are substitutes), the steady state is unstable, so unbounded growth is now possible.
} 
types. In particular to the labor spent in the cooperative. We regard the proportional sharing rule as meritocratic because it yields a proportion of consumption (relative reward) equal to the proportion of labor spent (relative merit).

\section{A Static Model of a Cooperative}

In this section we describe the main ingredients of our static analysis. Most of our concepts and assumptions are borrowed from standard economic theory.

There are $n$ workers that supply labor denoted by $l_{i}, i \in N=\{1,2, \ldots . n\}$. Let $l \equiv\left(l_{1}, l_{2}, \ldots, l_{n}\right)$, $l_{-i} \equiv\left(l_{1}, \ldots, l_{i-1}, l_{i+1}, \ldots, l_{n}\right), L \equiv \sum_{j=1}^{n} l_{j}$. There is a maximum quantity of labor that any worker can supply, $\bar{l}$.

Agents share a technology represented by a production function $X: \mathbb{R}_{+}^{n} \rightarrow \mathbb{R}_{+}$. The function $X$ is assumed to be concave, increasing and differentiable in $[0, \bar{l}]^{n}$ with $X(0)=0$.

Let $x_{i}$ be the consumption of $i$ and $x \equiv\left(x_{1}, x_{2}, \ldots, x_{n}\right)$. The pair $(x, l)$ is an allocation. An allocation $(x, l)$ is feasible if $\sum_{i=1}^{n} x_{i} \leq X(l)$ and $l_{i} \in[0, \bar{l}], i \in N$. The set of feasible allocations is denoted by $\mathcal{A}$.

Each worker $i$ has preferences over consumption and labor representable by a concave and differentiable utility function $U_{i}=U_{i}\left(x_{i}, l_{i}\right)$ which is strictly increasing in the first argument and strictly decreasing in the second argument. An economy is defined as a list of utility functions, $U \equiv\left(U_{1}, U_{2}, \ldots, U_{n}\right)$, satisfying the assumptions listed above. Let $\mathcal{E}$ be the set of all admissible economies.

The Pareto efficient solution, $\varphi^{E}: \mathcal{E} \rightarrow \mathcal{A}$ associates to each economy in the domain the set of Pareto efficient allocations for this economy.

These allocations are found by

$$
\max \sum_{i=1}^{n} \alpha_{i} U_{i}\left(x_{i}, l_{i}\right) \text { with }(x, l) \in \mathcal{A}
$$

for given $\left(\alpha_{1}, \alpha_{2}, \ldots, \alpha_{n}\right) \equiv \alpha$ such that $\alpha_{i} \geq 0$ and $\sum_{i=1}^{n} \alpha_{i}=1$. This is the maximization of a continuous function over a compact set and, hence, it has a solution by Weierestrass theorem. The program is concave and thus first order conditions give us the maximum. Assuming interiority, and denoting by $\lambda$ the Lagrange multiplier associated with the balance constraint, we have that

$$
\alpha_{i} \frac{\partial U_{i}\left(x_{i}, l_{i}\right)}{\partial x_{i}}=\lambda \text { and } \alpha_{i} \frac{\partial U_{i}\left(x_{i}, l_{i}\right)}{\partial l_{i}}=-\lambda \frac{\partial X(l)}{\partial l_{i}} \forall i \in N \text {. Thus, }
$$




$$
\frac{-\frac{\partial U_{i}\left(x_{i}, l_{i}\right)}{\partial l_{i}}}{\frac{\partial U_{i}\left(x_{i}, l_{i}\right)}{\partial x_{i}}}=\frac{\partial X(l)}{\partial l_{i}}, i \in N .
$$

Equation (2.1) says that the marginal rate of substitution between labor and consumption equals the marginal productivity of labor (which in our case is the marginal rate of transformation between labor and consumption). If labor is homogeneous, marginal productivities are identical so (2.1) implies the equalization of marginal rates of substitution between any pair of agents (a condition reminiscent of an exchange economy).

A Sharing Rule specifies the consumption allocated to each worker as a function of labor inputs. Formally, a sharing rule, $\mathbf{x}$, is a collection of differentiable functions $\left(\mathbf{x}_{1}, \mathbf{x}_{2}, \ldots, \mathbf{x}_{n}\right)$ with $\mathbf{x}_{i}: \mathbb{R}_{+}^{n} \rightarrow$ $\mathbb{R}_{+}, i \in N$, such that $\sum_{j=1}^{n} \mathbf{x}_{j}(l)=X(l), \forall l \in[0, \bar{l}]^{n}$. Well-known examples of sharing rules are:

$$
\begin{aligned}
\mathbf{x}_{i}^{P}(l) & =\frac{l_{i}}{L} X(l), \text { for all } i \in N \\
\mathbf{x}_{i}^{E}(l) & =\frac{1}{n} X(l), \text { for all } i \in N \\
\mathbf{x}_{i}^{\gamma}(l) & =\gamma \frac{l_{i}}{L} X(l)+(1-\gamma) \frac{1}{n} X(l), \gamma \in[0,1], i \in N .
\end{aligned}
$$

The family of sharing rules $\mathbf{x}^{\gamma}$ is parametrized by $\gamma$. If $\gamma=0, \mathbf{x}^{\gamma}$ is the egalitarian sharing rule and if $\gamma=1, \mathbf{x}^{\gamma}$ is the proportional sharing rule. The parameter $\gamma$ is a measure of how relative effort is valued and thus measures the degree of meritocracy which play an important role in our analysis. Another interested sharing rule is the Equal Benefit rule, in which each worker consumes according to her budget constraint of the Walrasian equilibrium with equal distribution of profits, Formally,

$$
\mathbf{x}_{i}^{E B}(l)=\frac{\partial X(l)}{\partial l_{i}} l_{i}+\frac{1}{n}\left(X(l)-\sum_{j=1}^{n} \frac{\partial X(l)}{\partial l_{j}} l_{j}\right), i \in N
$$

The interested reader can find in Moulin (1987) and Pfingsten (1991) other examples of sharing rules.

An efficient sharing rule, denoted as $\varphi^{\mathbf{x}}$, selects efficient allocations whose consumption allocated to each worker is determined by the sharing rule $\mathbf{x}$, formally:

$$
\varphi^{\mathbf{x} E}(U)=\left\{(x, l) \in \varphi^{E}(U) \mid x_{i}=\mathbf{x}_{i}(l), \text { for all } i \in N\right\}
$$

Corchón and Puy (2002) proved that $\varphi^{\mathbf{x} E}(U)$ is not empty whenever the sharing rule $\mathbf{x}$ is continuous. The proof is inspired by the proof of Negishi (1960) of the existence of a Walrasian 
Equilibrium. The sharing rules described above are all continuous, and hence, efficient sharing rules. This implies that any continuous sharing rule deemed as meritocratic, is compatible with efficiency since there are Pareto efficient allocations that belong to this rule.

\section{Efficiency, Meritocracy and Incentives in a Static Model}

We now turn our attention to the non-cooperative part of the problem. If labor contributions are

voluntary, we have a game in which the strategy space for each worker is $[0, \bar{l}]$ and agents receive the share of the total production dictated by a sharing rule, $x_{i}=\mathbf{x}_{i}(l)$ and $\mathbf{x}(l)=\left(\mathbf{x}_{1}(l), \mathbf{x}_{2}(l), \ldots, \mathbf{x}_{n}(l)\right)$. We refer to this game as the contribution game. Payoff functions are $U_{i}\left(\mathbf{x}_{i}(l), l_{i}\right), i \in N$. A Nash equilibrium of this game in normal form is a vector of strategies, $l^{*}$, such that

$$
U_{i}\left(\mathbf{x}_{i}\left(l^{*}\right), l_{i}^{*}\right) \geq U_{i}\left(\mathbf{x}_{i}\left(l_{1}^{*}, . ., l_{i}, . ., l_{n}^{*}\right), l_{i}\right) \text { for all } l_{i} \in[0, \bar{l}] \text {, for all } i \in N
$$

First order conditions of an interior Nash equilibrium are

$$
\begin{gathered}
\frac{\partial U_{i}\left(x_{i}, l_{i}\right)}{\partial x_{i}} \frac{\partial \mathbf{x}_{i}(l)}{\partial l_{i}}+\frac{\partial U_{i}\left(x_{i}, l_{i}\right)}{\partial l_{i}}=0, \text { or } \\
\frac{-\frac{\partial U_{i}\left(x_{i}, l_{i}\right)}{\partial l_{i}}}{\frac{\partial U_{i}\left(x_{i}, l_{i}\right)}{\partial x_{i}}}=\frac{\partial \mathbf{x}_{i}(l)}{\partial l_{i}}
\end{gathered}
$$

Comparing equations (2.1) and (3.1) we see that, in general, Nash equilibria are not Pareto efficient unless

$$
\frac{\partial X(l)}{\partial l_{i}}=\frac{\partial \mathbf{x}_{i}(l)}{\partial l_{i}}
$$

Assume that (i) all agents are identical; (ii) the sharing rule is a convex combination of the proportional and the equal share sharing rules, $\mathbf{x}^{\gamma}(l)$; and (iii) the production function is of CES form, $X(l)=\left(\sum_{i=1}^{n} l_{i}^{\rho}\right)^{\frac{r}{\rho}}$ with $\rho \leq 1$ and $0 \leq r \leq 1$, we have the following result.

Proposition 1. Under assumptions (i), (ii) and (iii), any Nash equilibrium of the contribution game yields efficient allocations if and only if $\gamma=r$.

Proof. First, under assumption (i), any Nash equilibrium, $l^{*}$, is symmetric. Which implies that $\mathbf{x}_{i}\left(l^{*}\right)=(1 / n) X\left(l^{*}\right)$. Furthermore, under assumption (ii),

$$
\frac{\partial \mathbf{x}_{i}\left(l^{*}\right)}{\partial l_{i}}=\frac{\gamma(n-1)}{n^{2} l_{i}^{*}} X\left(l^{*}\right)+\frac{1}{n} \frac{\partial X\left(l^{*}\right)}{\partial l_{i}}
$$


Thus, $\partial X\left(l^{*}\right) / \partial l_{i}=\partial \mathbf{x}_{i}\left(l^{*}\right) / \partial l_{i}$ if and only if

$$
\frac{\partial X\left(l^{*}\right)}{\partial l_{i}} \frac{(n-1)}{n}=\frac{\gamma(n-1)}{n^{2} l_{i}^{*}} X\left(l^{*}\right) .
$$

Or equivalently, if and only if,

$$
\frac{\partial X\left(l^{*}\right)}{\partial l_{i}} \frac{l_{i}^{*}}{X\left(l^{*}\right)}=\frac{\gamma}{n}
$$

Which under the assumption (iii) on the production function implies $\gamma=r$. Since second order conditions hold, any Pareto efficient Nash equilibrium implies $r=\gamma$. Conversely if $r=\gamma$ first order condition of efficiency and equilibrium coincide and since second order conditions hold, the result follows.

Sen (1966) proved a special case of this proposition when the product is homogeneous.

The intuitive reason why the sharing rule $\mathbf{x}^{\gamma}(l)$ works is that $\gamma$ measures the degree of responsiveness of production to inputs. The equalitarian sharing rule does not encourage effort as much as the proportional does because in the former the marginal return of effort is less than under the proportional rule. Thus, when output is not very responsive to efforts, to apply the proportional rule is not a good idea. But when output is very responsive to labor, efficiency requires a lot of work and to apply the proportional rule is a good idea. The question is whether this result is generalizable to economies where agents are different. Suppose first that there are a large number of workers and $X(L)=(L)^{r}$ with $r \leq 1$. In this case it is reasonable to assume that an individual, when taking her decision about her labor contribution, considers that the impact of this decision on total labor contribution is insignificant. Thus,

$$
\frac{\partial \mathbf{x}_{i}\left(l^{*}\right)}{\partial l_{i}}=L^{r} \gamma \frac{1}{L}
$$

Since for $X(L)=L^{r}$ we have that $\partial X\left(l^{*}\right) / \partial l_{i}=r L^{r-1}$, Nash equilibrium is compatible with efficiency if and only if $r=\gamma$.

The previous results are valid when workers are identical or the cooperative is large. When none of these two assumptions hold, Browning (1983) showed that the contribution mechanism described by Sen achieves efficiency only when the production function fulfills a separability property. Beviá and Corchón (2009) showed that when product is homogeneous, the contribution mechanism achieves efficiency only when the production function is a polynomial of, at most, degree $n-1$. We offer in this survey the analysis with two agents to give a flavor of the general result. We introduce 
some useful notation in what follows. Let

$$
R=\left\{l \mid \exists U \in \mathcal{E},(\mathbf{x}(l), l) \in \varphi^{E}(U)\right\} .
$$

$R$ is the set of input allocations that, given a sharing rule $\mathbf{x}$, yield efficient allocations for some economy. We refer to $l$ as an efficient input allocation. Define,

$$
R_{i}\left(l_{j}\right)=\left\{l_{i} \mid \exists U \in \mathcal{E},\left(x\left(l_{i}, l_{j}\right),\left(l_{i}, l_{j}\right)\right) \in \varphi^{E}(U)\right\} .
$$

$R_{i}\left(l_{j}\right)$ is the set of input contributions for $i, l_{i}$, such that $\left(l_{i}, l_{j}\right)$ is an efficient input allocation for some economy. Under the assumptions on the set of admissible economies, $R_{i}\left(l_{j}\right)$ is the interval $[0, \bar{l}]$, and $\forall l \in[0, \bar{l}]^{n}, \exists U \in \mathcal{E}$ such that $l$ is the unique efficient input allocation for $U$.

With this notation in hand, we can establish the following result which generalization for $N=$ $\{1, \ldots, n\}$ can be found in Beviá and Corchón (2009).

Proposition 2. Suppose $N=\{1,2\}$. If Nash equilibrium of the contribution mechanism yields efficient allocations in any $U \in \mathcal{E}$, the production function displays constant returns to scale.

Proof. Take any $U \in \mathcal{E}$ and consider a Pareto efficient allocation $\left(\mathbf{x}\left(l^{*}\right), l^{*}\right)$ such that $l^{*}$ is a Nash equilibrium. Thus,

$$
\frac{\partial \mathbf{x}_{i}\left(l^{*}\right)}{\partial l_{i}}=\frac{d X\left(l_{i}^{*}+l_{j}^{*}\right)}{d L}, \forall i \in N .
$$

The above equation holds in the interval $R_{i}\left(l_{-i}^{*}\right)$. Integrating on $\left[0, l_{i}\right]$ we get

$$
\mathbf{x}_{i}\left(l_{i}, l_{j}^{*}\right)=X\left(l_{i}+l_{j}^{*}\right)-Q_{i}, \forall l_{i} \in R_{i}\left(l_{j}^{*}\right), \forall i \in N
$$

where $Q_{i}$ depends on $l_{j}^{*}$. Since the above equation holds for all $l_{j} \in R_{j}\left(l_{i}\right), \forall j \neq i$,

$$
\mathbf{x}_{i}\left(l_{i}, l_{j}\right) \equiv X\left(l_{i}+l_{j}\right)-Q_{i}\left(l_{j}\right), \forall\left(l_{i}, l_{j}\right) \in R, \forall i \in N .
$$

Adding over $i$ and considering feasibility we obtain

$$
X\left(l_{i}+l_{j}\right) \equiv Q_{i}\left(l_{j}\right)+Q_{j}\left(l_{i}\right), \forall l \in R .
$$

(see Browning (1983)). Consider now the vectors $\left(l_{i}, 0\right),\left(0, l_{j}\right)$, and $(0,0)$. Since $X(0+0)=0$, $Q_{i}(0)+Q_{j}(0)=0$, and using $(3.3)$

$$
X\left(l_{i}+l_{j}\right)-X\left(l_{i}\right)-X\left(l_{j}\right)=0
$$


The solution of (3.4) is a polynomial of, at most, degree 1 (Aczel (1966) pp. 129-130). Thus, the production function display constant returns to scale.

When $N=\{1,2\}$, to distribute the total output in a way that each worker receives her marginal contribution to the total production represents a sharing rule whenever the production function has constant returns to scale. Formally, this sharing rule is defined as

$$
\mathbf{x}_{i}(l)=X\left(l_{i}+l_{j}\right)-X\left(l_{j}\right)
$$

We refer to $\mathbf{x}$ as the Incremental Sharing Rule.

Proposition Let $N=\{1,2\}$ and suppose that the production function, $X$, displays constant returns to scale. All Nash equilibria associated with an anonymous sharing rule are efficient if and only if the sharing rule is the incremental sharing rule.

Proof. Suppose that Nash equilibrium are efficient. By anonymity and feasibility, $\mathbf{x}_{i}(0,0)=$ $\mathbf{x}_{j}(0,0)=0$. As we have shown in Proposition 2, the sharing rule should satisfy (3.2), which together with anonymity implies that $Q_{i}(0)=Q_{j}(0)=0$. Given $l=\left(l_{i}, l_{j}\right)$, by $(3.3), X\left(l_{j}\right)=Q_{i}\left(l_{j}\right)$, therefore $x_{i}\left(l_{i}, l_{j}\right)=X\left(l_{i}+l_{j}\right)-X\left(l_{j}\right)$. Thus, the sharing rule is the incremental sharing rule. Finally, notice that the allocation yielded by Nash equilibrium of the contribution game associated to the incremental sharing rue is efficient because the incremental sharing rule equalizes social and private gains.

The previous result may look like negative but this is only because we assume 2 workers only. Beviá and Corchón (2009) proved the previous result for arbitrary $n$. And in this case, the production function must be a polynomial of at most degree $n-1$. Given that any continuous function in a compact interval can be approximated by a polynomial this suggest that the incremental sharing rule implements, approximately, an efficient solution when the number of workers is high. For $N=\{1, \ldots, n\}$, this rules is defined as

$\mathbf{x}_{i}^{I}(l)=X(L)-(n-1) X\left(L_{-i}\right)+\frac{n-1}{2} \sum_{k \neq i} X\left(L_{-i k}\right)-\frac{n-1}{3} \sum_{k, h \neq i} X\left(L_{-i k h}\right)+. .+(-1)^{n-1} \sum_{j \neq i} X\left(l_{j}\right)$

where $L_{-i} \equiv \sum_{j \neq i} l_{j}, L_{-i k}=\sum_{j \neq i, j \neq k} l_{j}, L_{-i k m}=\sum_{j \neq i, j \neq k, j \neq m} l_{j}$ and so on. The sharing rule is well define whenever $X$ is a polynomial of at most degree $n-1$.

Note that

$$
\mathbf{x}_{i}^{I}(l)-\mathbf{x}_{i}^{I}\left(0, l_{-i}\right)=X(L)-X\left(L_{-i}\right) .
$$


This rule has two parts. One part awards each worker with her marginal product as it happens with the celebrated Vickrey-Clark-Groves (VCG) mechanisms. The other part is composed by terms that depend on the contributions of other agents. Despite the complex analytical form,, the Incremental Sharing Rule is quite simple. It demands the equalization between private gain in consumption of $i$ and public gain in aggregate output for each variation of the labor supplied by $i^{13}$

When the assumption that the production function is polynomial is not a reasonable approximation, we have to rely on the full force of implementation theory. In this framework, each worker has a message space indicating the kind of communication that she is allowed to make. For each profile of messages there is an outcome. The planner enforces allocations but she does not know the preferences of agents. Corchón and Puy (2002) provided a simple mechanism that, when there are at least three workers, implements in Nash equilibrium every efficient sharing rule. ${ }^{14}$ This means that every message that constitutes a Nash equilibrium yields the desired allocations and that the set of Nash equilibria is not empty. In their mechanism, people are arranged in a circle and each worker proposes the amount of input supplied by him and the worker next to him. Here we reproduce verbally how the mechanism works. For the technical details see Corchón and Puy (2002) Theorem 2. The mechanism has three rules:

1. When the amount of input proposed by each worker coincides with the amount suggested by her monitor, the mechanism distributes the output according to the sharing rule.

2. When there are, at most, two consecutive workers whose proposals differ from what was proposed for them, the worker with the lowest index (the dissident) has the right to choose an allocation in a certain budget set that is only profitable if she has deviated from a non efficient allocation. Since a deviation can only happen if the monitor of the dissident has tried to fool the mechanism, the monitor of this worker is severely fined: she gets zero consumption and has to contribute with the maximum amount of labor. All other workers obtain some arbitrary bundle.

3. For any other message, the mechanism divides workers into two groups: the ones that consume

\footnotetext{
${ }^{13}$ Moulin (2010) provided a similar characterization of the incremental sharing rule (the residual ${ }^{*}$ mechanism as Moulin called it) in the context of cost sharing. Formally, one result does not imply the other or viceversa because the two games are not the same. In Beviá and Corchón (2009), the game is a contribution game associated to an output sharing problem and in Moulin's case it is a demand game associated to a cost-sharing game. Leroux (2008) has proved that these two games are different.

${ }^{14}$ All the previos contributions focused on the implementation of a single sharing rule, see Corchón and Puy (2002).
} 
but do not work, and the ones that work but do not consume. Clearly to announce an efficient allocation proposed by the sharing rule is a Nash equilibrium, because deviations can only punish the deviator. And any Nash equilibrium yields the desired allocations because if it occurs in the first case a deviator can obtain a larger utility by deviating and there is no equilibrium in the second and the third case since there is always a worker who can deviate and improve her payoff.

Clearly to announce the contributions that are efficient is an equilibrium. The converse, i.e. to prove that all Nash equilibria yield the desired allocation is harder, see Corchón and Puy (2002) for details.

\subsection{Sabotage}

In this section we broaden the scope of incentive theory by considering situations in which agents can sabotage production by destroying other agents' inputs. The input supplied by a worker reflects now her effort and the sabotage done by others on this worker. In Beviá and Corchón (2006) is assumed that sabotage is undetectable because the effort and the technology of sabotage are not contractible. They show that sabotage might arise in the framework of cooperative production, in which the output is entirely distributed to workers.

In order to formally analyze the possibilities of sabotage, we introduce some basic notation and small modifications in the cooperative production model described in the previous section. We focus in the survey on an specific case with identical agents, with a CES production function and the family of sharing rules generated as a convex combination of the proportional and the egalitarian one. The analysis of a more general case can be found in Beviá and Corchón (2006).

The quantity of labor time that each worker can supply is fixed and equal to $T$. An worker $i$ can divide her working time between productive labor, $l_{i}^{P}$, and sabotage activities. Let $l_{i j}$ be the quantity of labor allocated by $i$ to sabotage the input of worker $j$. Thus, $T=l_{i}^{P}+\sum_{j \neq i} l_{i j}$. The input provided by worker $i, I_{i}$, depends on her own productive effort, $l_{i}^{P}$, and the amount of time devoted by the remaining agents to sabotaging the input of $i$, i.e. $I_{i}=I\left(l_{i}^{P}, l_{1 i}, \ldots l_{(i-1) i}, l_{(i+1) i}, \ldots l_{n i}\right)$ where $I\left(\right.$ ) is a $\mathcal{C}^{1}$ function such that

$$
\frac{\partial I\left(l_{i}^{P}, l_{1 i}, \ldots l_{(i-1) i}, l_{(i+1) i}, \ldots l_{n i}\right)}{\partial l_{i}^{P}}>0 \text { and } \frac{\partial I\left(l_{i}^{P}, l_{1 i}, \ldots l_{(i-1) i}, l_{(i+1) i}, \ldots l_{n i}\right)}{\partial l_{j i}}<0 \text { for all } l_{j i}
$$

Suppose a constant elasticity of substitution production function, $X\left(I_{1}, \ldots, I_{n}\right)=\left(\sum_{i=1}^{n}\left(I_{i}\right)^{\rho}\right)^{\frac{r}{\rho}}$ with 
$\rho \leq 1$ and $r \leq 1$. The sharing rule is $\mathbf{x}^{\gamma}$ that now depends on inputs, that is,

$$
\mathbf{x}_{i}^{\gamma}\left(I_{1}, . . I_{n}\right)=\left(\gamma \frac{I_{i}}{\sum_{j=1}^{n} I_{j}}+(1-\gamma) \frac{1}{n}\right) X\left(I_{1}, \ldots, I_{n}\right) .
$$

The sabotage game is described as follows: for each worker $i$, a strategy is the time devoted to sabotage activities, i.e. the vector $l_{i}=\left(l_{i 1}, . ., l_{i(i-1)}, l_{i(i+1)}, . ., l_{i n}\right)$. Time devoted to productive activities is determined by the constraint $l_{i}^{P}=T-\sum_{j \neq i} l_{i j}$. For each worker $i$, given a vector of strategies $\left(l_{i}, l_{-i}\right)$, the payoff function is given by

$$
\begin{aligned}
\pi_{i}\left(l_{i}, l_{-i}\right) & \equiv \mathbf{x}_{i}^{\gamma}\left(I_{1}, . . I_{n}\right) \text { where } \\
I_{j}\left(l_{i}, l_{-i}\right) & \equiv I\left(T-\sum_{j \neq i} l_{j i}, l_{1 j}, \ldots l_{(j-1) j}, l_{(j+1) j}, \ldots l_{n j}\right), j \in N
\end{aligned}
$$

A Nash equilibrium of the sabotage game, denoted by $N E$, is a vector of strategies $\left(l_{1}, . ., l_{n}\right)$ such that for all worker $i, \pi_{i}\left(l_{i}, l_{-i}\right) \geq \pi_{i}\left(l_{i}^{\prime}, l_{-i}\right)$ for all $l_{i}^{\prime}$.

Beviá and Corchón (2006) established the conditions under which the existence of a Nash equilibrium is guarantee. In this survey we concentrate on the necessary conditions that guarantee that there is no sabotage in equilibrium under the above specifications.

Let

$$
M \equiv-\frac{\frac{\partial I_{j}(0,0)}{\partial l_{i j}}}{\frac{\partial I_{i}(0,0)}{\partial l_{i}^{P}}} .
$$

$M$ is a measure of the relative impact of change in inputs induced by a re-allocation of labor time of $i$ from productive activities to sabotage activities evaluated at the point of zero sabotage. Thus, $M$ is a measure of the power of destruction versus production capabilities. Abusing language, we will say that $M$ is a measure of the possibilities of destruction. From our assumptions it follows that $M>0$. Furthermore, because of our symmetry assumption on the input functions, $M$ is independent of $i$ and $j$.

With this notation in hand, we can give the following result:

Proposition 3. If zero sabotage is a Nash equilibrium of the sabotage game, then $(M+1-n) \gamma \leq$ $r(M+1)(n-1)$.

Proof. The proof follows from the observation that if no worker has incentive to sabotage when all other agents do not sabotage, it must be that for all $i, j, \partial \pi_{i}(0,0) / \partial l_{i j} \leq 0$ which, under the assumptions imposed in this section implies that $(M+1-n) \gamma \leq r(M+1)(n-1)$. 
The condition $(M+1-n) \gamma \leq r(M+1)(n-1)$ always hold when the cooperative is large or when the possibilities of destruction via sabotage $(M)$ are small because in this case the left hand side of the inequality is negative and the right hand side is positive. But when $M+1-n>0$ this inequality puts an upper bound to the degree of meritocracy that is compatible with no sabotage. In this case a small $r$ is bad to avoid sabotage because $r$ is an inverse measure of congestion. When $r$ is small, efforts do not translate into output easily so sabotage is tempting.

\section{Voting and Cooperation in a Static Model}

Many decisions in real cooperatives are taken by voting, sometimes delegating the decision in a democratically chosen Board of Directors (BOD). In this section we analyses the class of sharing rules that will be chosen if members of the cooperative have the right to vote on her preferable choice. To make the problem tractable, we assume that there are an odd number of workers in the cooperative and workers preferences are represented by a quasilinear utility function, $U_{i}\left(x_{i}, l_{i}\right)=$ $x_{i}-v_{i}\left(l_{i}\right)$, where $v_{i}$ is strictly increasing and strictly convex for all $i \in N$. We also assume that workers have to choose a sharing rule in the class $\mathbf{x}^{\gamma}$ taking into account that her labor contribution, given the chosen rule, has to be the efficient one, that is $\left(\mathbf{x}^{\gamma}(l), l\right) \in \varphi^{\mathbf{x}^{\gamma} E}(U)$.

Given the quasilinear assumption, an efficient allocation, $(\hat{x}, \hat{l})$, satisfies the following condition:

$$
\frac{\partial X(\hat{l})}{\partial l_{i}}=\frac{\partial v_{i}\left(\hat{l}_{i}\right)}{\partial l_{i}} \text { for all } i \in N
$$

Since preferences are strictly convex, condition (4.1) determines a unique vector $\hat{l}$ which is independent on the degree of meritocracy $\gamma$. The preferred $\gamma$ for each worker will depend on

$$
\frac{\partial U_{i}\left(\mathbf{x}_{i}^{\gamma}(\hat{l}), \hat{l}_{i}\right)}{\partial \gamma}=X(\hat{l})\left(\frac{\hat{l}_{i}}{\sum_{i=1}^{n} \hat{l}_{i}}-\frac{1}{n}\right) .
$$

For all agents $i$ such that $\hat{l}_{i}>\left(\sum_{i=1}^{n} \hat{l}_{i}\right) / n,(4.2)$ is positive and therefore, their preferred level of meritocracy is $\gamma=1$. For those agents such that $\hat{l}_{i}<\left(\sum_{i=1}^{n} \hat{l}_{i}\right) / n,(4.2)$ is negative and therefore, their preferred level of meritocracy is $\gamma=0$. Thus, the chosen sharing rule will be either $\gamma=1$ or $\gamma=0$ depending on the labor contribution of the median voter. If it is above the average total labor, the sharing rule will be meritocratic otherwise will be egalitarian. We summarize this result in the following proposition. 
Proposition 4. Suppose preferences are quasilinear and workers have to choose by majority voting a sharing rule in the class $\mathbf{x}^{\gamma}$. Let $\left(\mathbf{x}^{\gamma}(\hat{l}), \hat{l}\right) \in \varphi^{\mathbf{x}^{\gamma} E}(U)$, and let $\hat{l}_{\text {med }}$ the labor contribution of the median voter. Then, if $\hat{l}_{\text {med }} \geq\left(\sum_{i=1}^{n} \hat{l}_{i}\right) / n$ the sharing rule will be meritocratic, $\gamma=1$, otherwise will be egalitarian, $\gamma=0$.

Note that if $\hat{l}_{\text {med }}=\left(\sum_{i=1}^{n} \hat{l}_{i}\right) / n$, the median voter is indifferent between any sharing rule. We have broken ties in favor of meritocracy.

In Corchón and Puy (1998) this analysis is extended by considering the class of sharing rules that are a convex combinations of the Proportional, the Egalitarian and the Equal Benefit sharing rules. They first proved that if we want not only efficient sharing rules but also individually rational sharing rules (each worker gets at least her reservation value, that they suppose in their paper that is zero), the weight of the egalitarian sharing rule has to be zero. This is because they show an example in which efficiency forces an able worker to exert hard effort and to a non-able worker to exert very little effort. Given that consumption is equally shared among them, the able worker is better off by neither consuming, nor working. Having discarded egalitarianism, agents have to choose, by majority voting, a sharing rule in the class formed by convex combinations of the Proportional and the Equal Benefit sharing rules. They prove that preferences of the agents are single peak with the peak in the extremes, that is, either the most preferred sharing rule is the Proportional or the Equal benefit. These preferences depends for each worker (like in Proposition 4) on the comparison between $i$ efficient labor contribution and the average efficient labor. ${ }^{15}$

The previous result suggest that, if there were several potential cooperatives and workers could move freely among them, they would cluster in cooperatives with similar productivities with a sharing rule either proportional or egalitarian. Barberá, Beviá and Ponsati (2015) take a closer look to a situation in which the set of workers are partitioned in different cooperatives. And once the cooperative is organized, members choose their sharing rule by majority voting among the class of sharing rules $\mathbf{x}^{\gamma}$. They focus on the notion of Stability which requires that no group of agents has incentives to organize a different cooperative than the one they belong in the partition. They assume that the production function has constant returns to scale but a minimal number of workers $\nu$ are needed for the cooperative to be productive. As before, preferences are quasilinear. As we

\footnotetext{
${ }^{15}$ A result identical to Proposition 4 is obtained by replacing the Egalitarian sharing rule by the Equal Benefit sharing rule.
} 
will see, they show that meritocratic and egalitarian cooperatives can coexist and that stability do not imply a segregation of groups of similar productivity.

As in Corchón and Puy (1991), the efficient contribution of labor for each worker (which is determined by (4.1)) is independent on the other agents contributions. Thus, we can describe a cooperative by a vector of efficient labor $l=\left(l_{1}, \ldots, l_{n}\right)$. Note that since the group of agents that will form a cooperative is endogenous, we can not make the assumption that there is an odd number of agents. So, it could be that for some cooperatives there are several median voters. In this case ties are broken in favor of the worker with the highest productivity. To simplify our presentation suppose that $\nu=N / 2$ and there are three type of agents: high, medium and low productive agents $i \in\{h, m, l\}$, with productivity $l_{h}, l_{m}$, and $l_{l}$ respectively, with $l_{h}>l_{m}>l_{l}$. The sets $H, M$, and $L$ denote the sets of all high, medium and low type agents respectively and $n_{H}, n_{M}$ and $n_{L}$ denote the cardinality of these sets. A class of societies that are key for the analysis of stable partitions is the maximally mixed meritocratic societies formally defined as the class of societies $(N, \nu, l)$ such that $0<n_{H}<v / 2,0<n_{L} \leq v / 2$, and $\left(l_{h}+l_{m}+n_{L} l_{l}\right) /\left(n_{L}+2\right) \leq l_{m}$. In this class of societies, it is always possible to construct a meritocratic cooperative of cardinality $\nu$ with agents of all three types. The following example illustrates this point.

Example 1. (Barberá, Beviá and Ponsatí (2015)) A society with a stable partition with nonsegregated groups where different sharing rules coexist.

Let $N=\{1,2,3,4,5,6,7,8,9,10\}, \nu=5$, and $l=(100,100,75,75,75,75,75,75,75,45)$. Let $G_{1}=$ $\{1,3,4,5,10\}$ and $G_{2}=\{2,6,7,8,9\}$. Note that $G_{1}$ will choose a meritocratic sharing rule and $G_{2}$ will choose an egalitarian one. Let us see that the partition $\pi=\left(G_{1}, G_{2}\right)$ is stable. Note that the medium type agents in $G_{2}$ can only improve if a high type is added to the coalition or if a medium type is substituted by a high type. But since the other high type not in $G_{2}$ is already in a meritocratic coalition, she does not have incentives to form the potential blocking coalition. The two high types cannot be together in a meritocratic coalition, and any other worker needs high types to improve. That implies that $\pi$ is a stable partition. Note that high and medium productivity agents are split between the two coalitions.

The following result, characterize the kind of cooperatives that can be formed in equilibrium

Proposition 5. (Barberá, Beviá and Ponsatí (2015))

(a) In three type societies where $v=n / 2$, stable partitions always exist. 
(b) If societies are maximally mixed meritocratic, stability implies non-segregation and one of the cooperatives is meritocratic.

(c) If societies are not maximally mixed meritocratic, then the segregated partition where a coalition of most productive agents of size $v$ is formed, and the rest of agents gather together in a second coalition is always stable.

Thus, contrarily to the conjecture by Corchón and Puy (1991), when workers can form and quit cooperatives, stability could imply non segregated cooperatives populated by agents with different productivities.

These models have several shortcomings, among them the assumption of quasi-linearity in consumption. Torregrosa-Montaner (2017) has analyzed the case of a continuum of workers with identical Cobb-Douglas utility functions but different endowments and $X(L)=L^{r}$. In his model, effort and consumption depend on endowments. As before, workers vote on the level of meritocracy $\gamma$. He finds that low-wealthy (resp. high-wealthy) cooperatives choose degrees of meritocracy below (resp. above) the optimal. Thus, poor (resp. rich) workers are willing to sacrifice efficiency for (resp. lack of) redistribution. When the distribution of wealth is symmetric, these two forces compensate each other and voting yields the efficient value of meritocracy, namely $r=\gamma$.

Summing up, voting, whatever other advantages it has, does not guarantee efficiency. On the contrary, the results that we surveyed point out that voting is likely to introduce biases in the degree of meritocracy and, when agents can form different cooperatives, in the composition and the number of cooperatives.

\section{Dynamics. Voting, Unemployment and Exploitation}

So far we have consider one period models. In this section we focus attention on a cooperative in which there is entry or exit in each period.

Beviá, Corchón and Romero (2016) have studied the dynamics of a cooperative when initial members of the cooperative decide both, whom to invite to join and the sharing rule applied in the cooperative among the class $\mathbf{x}^{\gamma}$. The cooperative and workers last for a countable infinite number of periods $\tau=(0,1, \ldots t, \ldots)$. In each period $\tau$, decisions to invite and to share the output are taken by plurality. We retain the assumptions made in the second part of Section 4: the production function has constant returns to scale, preferences are quasilinear and each worker in the cooperative has to 
contribute with the efficient labor. Following the analysis presented in the previous section, under those assumptions we can identified an economy with a vector $l$. Additionally, it is assume that there is only three type of agents, $i \in\{h, m, l\}$, with productivity $l_{h}, l_{m}$, and $l_{l}$ respectively, with $l_{h}>l_{m}>l_{l}$. The sets $H, M$, and $L$ denote the sets of high, medium and low type agents and $n_{H}^{\tau}$, $n_{M}^{\tau}$ and $n_{L}^{\tau}$ denote the cardinality of these sets at period $\tau$ that includes the new hirings. Assuming that the outside option of any worker is sufficiently low, all agents invited to join accept and no one wants to leave the cooperative. For simplicity, at each period $\tau$ only one worker is invited and it is assume that the pool of outsiders always contains agents from the three types. Beviá, Corchón and Romero (2016) studies the Markov Perfect Equilibria (MPE) of this dynamic game where the state at $\tau$ is the number of insiders of each type at $\tau-1$. Notice that at each period $\tau$, the insiders in $\tau-1$ decide on the sharing rule as in Proposition 4. Thus, high productivity agents always prefer the meritocratic sharing, low productivity agents always prefer the egalitarian sharing, and for medium productivity agents will depend on her labor contribution compared with the average contribution. Let $\delta$ be the discount factor which indicates how much agents care about the future.

It is important for the analysis of the dynamic equilibrium to identify at each period which is the dominant class. A group of a certain productivity is the dominant class at $\tau$ if it represents more than half of the population of insiders at $\tau-1$. Note that if a group is dominant, decisions are going to be chosen according to the preferences of this group. If there is no dominant group at $\tau$, Beviá, Corchón and Romero (2016) show that, for a sufficiently large $\delta$, the decisive group at $\tau$ is the group of medium productivity agents. The main result in Beviá, Corchón and Romero (2016) is summarized in the following proposition.

Proposition 6. (Beviá, Corchón and Romero (2016)). There exist $\bar{\delta}>0$ such that for all $\delta \geq \bar{\delta}$, (i) In any MPE if $H$ is the dominant class at period $\tau$, then $H$ is dominant at all subsequent periods and meritocracy will be the sharing rule.

(ii) In any MPE if $M$ is the decisive group at period $\tau$, then $M$ will be the decisive type at any subsequent period and eventually, egalitarianism will be the sharing rule.

(iii) In any MPE if $L$ is the dominant class at period $\tau$, then eventually egalitarianism will be the sharing rule although they will eventually loose political power in favor of the medium class.

An important point of this analysis is that, when insiders take the decision on who to invite, they have to take into account the consequences of this invitation in the future choices of the 
sharing rule. Clearly, if high types are the majority, the chosen sharing rule is the meritocratic one, and they will keep the majority by inviting high types. Given the constant returns assumption on the production function, they are indifferent among inviting agents from any type whenever the meritocratic sharing rule is the outcome of the future organizations. But in this case, Beviá, Corchón and Romero (2016) assume that ties are broken by choosing always the highest productivity worker. ${ }^{16}$ If low types are the majority, the sharing rule will be the egalitarian one, and they want to keep it this way in future organizations. Of course, they can do that by inviting only low types, but, whenever they can guarantee that the egalitarian sharing rule is going to be the outcome, they will prefer to increase total production. Thus why, at some point, they will prefer to give the power to the medium class (provided that the medium class also prefer the egalitarian sharing rule). Finally, if medium types are the decisive group, they will never hire low types, but they can hire high types whenever they do not risk their decisive power. And output will be share eventually in a egalitarian way.

Proposition 6 has important implications about the long run composition of a cooperative. In particular, high quality organizations (those with a high type dominant class) tend to persist over time. But organizations that are formed initially by other groups, never achieve full excellency. Those results are formally summarized in the following Corollary where $\left(m_{H}^{\tau}, m_{M}^{\tau}, m_{L}^{\tau}\right)$ is the proportion of insiders of type $H, M$ and $L$ respectively at time $\tau$.

Corollary 1. (Beviá, Corchón and Romero (2016)) For a $\bar{\delta}$ sufficiently close to 1 if the dominant class at $\tau=0$ is $H$, then $\lim _{\tau \rightarrow \infty}\left(m_{H}^{\tau}, m_{M}^{\tau}, m_{L}^{\tau}\right)=(1,0,0)$. In any other case $\lim _{\tau \rightarrow \infty}\left(m_{H}^{\tau}, m_{M}^{\tau}, m_{L}^{\tau}\right)=$ $(0.5,0.5,0)$.

In this model, the long run behavior of organizations is totally determined by the initial conditions and it is never a cooperative populated by low productivity workers only. But there are models of dynamic organizations in which this conclusion does not hold. In Sobel (2001) standards of admission and the average quality of incumbents rise or fall without any bound (which is impossible here because we only have three types). In Corchón (2005) there is free entry to the cooperative. A boss for a single period is chosen randomly among the insiders. And who is the boss, matters because a talented boss improves the productivity of all workers. In this model, the degree

\footnotetext{
${ }^{16}$ This is because under non constant returns, to introduce a high productivity worker would be preferrable. It is only because the assumption of constant returns that indifference among types arise.
} 
of meritocracy is the weight given by the lottery to choose a talented boss. A good boss may attract many low productivity workers whose life is less miserable under an able command than under their own. This is the Umbrella Effect that may help to explain why able bosses might be surrounded by mediocre people, without assuming that able leaders do not like potential challengers around. Thus the outcome of a policy of excellence in choosing leaders might be a cooperative populated by low productivity workers. In this model, when the cooperative is not populated by identical agents, the average productivity of the cooperative may cycle.

\section{Dynamics. Meritocracy and Accumulation of Human Capital}

The previous work studied how cooperatives may change due to the changes in the quantity of the existing or the potential labor force. What about changes in the quality of actual workers? Beviá and Corchón (2016) presented a dynamic model in which output is produced by human capital which, in turn, is produced by inheritance (say, the cultural level of your family) and effort (your grades). Thus your effort producing today human capital has long run effects in subsequent periods. The claim to output is not longer pure labor but human capital.

Formally, there are $n$ families with a member alive in each period. At period $t$, and individual from a family $i$ receives the capital $H_{i}^{t-1}$ (accumulated by the previous generation) and has a labor

endowment $\omega>1$. Each individual produces her own capital from the inherited capital, $H_{i}^{t-1}$ and her labor, $l_{i}^{t} \in[0, \omega]$, such that $H_{i}^{t}=H_{i}^{t-1} l_{i}^{t}$. Let $H^{t}=\sum_{j=1}^{n} H_{j}^{t}$ be the aggregate capital at period $t$. At $t=0, H_{i}^{0}=H_{j}^{0}$ for all families $i, j$. At each period $t$, the consumption good is produced using capital as input according to the production function $X\left(H^{t}\right)=\left(H^{t}\right)^{r}$. The consumption of an individual from a family $i$ is given by

$$
c_{i}^{t}=\left(H^{t}\right)^{r}\left(\gamma \frac{H_{i}^{t}}{H^{t}}+\frac{1-\gamma}{n}\right), \gamma \in[0,1],
$$

and her utility is represented by a Cobb-Douglas function of the form

$$
U_{i}\left(c_{i}^{t}, \omega-l_{i}^{t}\right)=(\beta+1)\left(c_{i}^{t}\right)^{\frac{\beta}{\beta+1}}\left(\omega-l_{i}^{t}\right)^{\frac{1}{\beta+1}}, \beta>0
$$

In what follows we assume that $n$ is large and when individuals take their decisions on her labor contribution, they take $H^{t}$ as given. We also assume that workers do not care about future 
generations. The maximization problem of worker $i$ is

$$
\begin{aligned}
& \operatorname{Max}_{\left(c_{i}^{t}, l_{i}^{t}\right)} U_{i}\left(c_{i}^{t}, \omega-l_{i}^{t}\right) \\
& \text { s.t. } c_{i}^{t}=\left(H^{t}\right)^{r}\left(\gamma \frac{H_{i}^{t}}{H^{t}}+\frac{1-\gamma}{n}\right) \\
& l_{i}^{t} \in[0, \omega]
\end{aligned}
$$

The solution to the above maximization problem is given by the first order conditions, which simplifying, can be written as

$$
l_{i}^{t}=\omega-\left(\frac{\left(H^{t}\right)^{1-r}}{\beta \gamma H_{i}^{t-1}}\right) c_{i}^{t} .
$$

Given that all individuals are identical, in equilibrium $H_{i}^{t-1}=H_{j}^{t-1} ; H_{i}^{t}=H_{j}^{t} ; H^{t-1}=n H_{i}^{t-1}$; $H^{t}=n H_{i}^{t}$. Thus, $l_{i}^{t}=H_{i}^{t} / H_{i}^{t-1}=H^{t} / H^{t-1}$, and $c_{i}^{t}=\left(H^{t}\right)^{\gamma} / n$, which implies that

$$
\frac{H^{t}}{H^{t-1}}=\frac{\omega \beta \gamma}{1+\beta \gamma}
$$

and the growth rate of human capital is

$$
g^{t}=\frac{H^{t}-H^{t-1}}{H^{t-1}}=\frac{\omega \beta \gamma}{1+\beta \gamma}-1
$$

The growth rate is constant, and it is positive iff $\beta \gamma(\omega-1)>1$. It increases with labor endowments (so cooperatives rich in endowments grow faster than cooperatives poor in endowments); in the taste of consumption (so consumerism is good for growth because incentives hard work) and the degree of meritocracy (meritocracy encourages hard work).

Beviá and Corchón (2016) extend this model in two directions. Firstly, they show that with a CES utility function the growth rate is no longer constant. In particular, if the elasticity of substitution between consumption and labor is smaller than one, a stable steady state exist. The capital at the steady state is increasing with the level of meritocracy. If consumption and leisure are substitutes, capital at the steady state depends negatively on meritocracy and the taste of consumption. This is because the steady state is unstable. Thus, given the initial stock of capital, more meritocracy or more appreciation for consumption may impulse the economy from negative to positive growth.

Secondly, keeping the Cobb-Douglas assumption, they introduce a concern for future generations. Each generation discount the welfare of the following generation by a factor of $\delta, \delta^{2}, \ldots$ etc. where $\delta<1$. They show that for a sufficiently small $\delta$, there is a solution to the intertemporal maximization problem in which the growth rate is constant and larger than the corresponding rate 
in the zero discounting case. This is due to the fact that when future counts, there are more incentives to invest in capital which in turn stimulates growth. The role of meritocracy is identical to the case $\delta=0$. Meritocracy encourages work which produces human capital, which makes the economy grow. For $\delta>0$ returns to scale does play a role. The larger they are, the larger is the growth rate. Finally they show that the optimal degree of meritocracy is reached, as in the cases showed in Section 3, when $r=\gamma$.

\section{Conclusion}

Our main conclusion is that meritocracy is compatible with incentives and efficiency only in the case in which we can use the full force of implementation theory. But in presence of sabotage, this compatibility is problematic. Egalitarianism is sabotage-free but may yield individual rationality problems. And in contribution mechanisms, meritocracy must be tempered with egalitarianism in shares that depend on the elasticity of output with respect to labor. Finally voting introduces distortions because workers are prepared to vote inefficient schemes that are stacked in their favour.

In our opinion, the theoretical work surveyed here present a basis to study how issues of meritocracy, efficiency, incentives and voting in cooperatives are coped with in the real world of cooperatives. And how the problems faced by cooperatives might be lessen by the theoretical insights here presented.

\section{References}

[1] Aczel, J. (1966), Lectures on Functional Equations and their Applications, Academic Press, NY.

[2] Barberá, S.,C. Beviá, and C. Ponsatì (2015). "Meritocracy, Egalitarianism and the Stability of Majoritarian Organizations". Games and Economic Behavior, 31, 237-257.

[3] Beviá, C. and L. Corchón (2006). "Rational Sabotage with Heterogeneous Agents". The B.E. Journals in Theoretical Economics, Topics. 6, 1.

[4] Beviá, C. and L. Corchón (2009)."Cooperative Production and Efficiency". Mathematical Social Sciences, 57, 2, 143-154. 
[5] Beviá, C. and L. Corchón (2017)."Growth in Illyria: The Role of Meritocracy in the Accumulation of Human Capital". Mathematical Social Sciences, in press.

[6] Beviá, C., L. Corchón and A. Romero-Medina (2016). "Relinquinshing Power, Exploitation and Political Unemployement in Democratic Organizations". Social Choice and Welfare, in press.

[7] Browning, M.J. (1983), "Efficient Decentralization with a Transferable Good." The Review of Economic Studies, 50, 2, 375-381.

[8] Corchón, L. (2005). "Monk business: An example of the dynamics of organizations". Social Choice and Welfare, 24, 1-14.

[9] Corchón, L. (2017) "The Theory of Implementation: What Did We Learn?". Encyclopedia of Complexity and Systems, Editor Meyers, Robert A. Springer, first edition, May 2009.

[10] Corchón, L. and S. Puy (1998)."Individual Rationality and Voting in Cooperative Production." Economics Letters 59, 83-90.

[11] Corchón, L. and S. Puy (2002). "Existence and Nash Implementation of Efficient Sharing Rules for a Commonly Owned Technology.". Social Choice and Welfare,19, 2, 369-379, 2002.

[12] Hart, Oliver (1979). "On Shareholder Unanimity in Large Stock Market Economies." Econometrica 47, 1057-1083.

[13] Kang, S. (1988). "Fair distribution rule in a cooperative enterprise". Journal of Comparative Economics, 12, 1, 89-92.

[14] Kremer, M. (1997). "Why are worker co-operatives so rare?". NBER Working Paper: 6118.

[15] Lazear, E. P. (1989). "Pay Equality and Industrial Politics," Journal of Political Economy, 97 (3), 561-580.

[16] Leroux, J. (2008), "Profit sharing in unique Nash equilibrium: Characterization in the twoagent case." Games and Economic Behaviour 62, 558-572.

[17] Moulin, H. (1987) "Equal or proportional division of a surplus, and other methods" International Journal of Game Theory, 16, 161-186. 
[18] Moulin, H. (2010). "An efficient and almost budget balanced cost sharing method". Games and Economic Behavior 70, 1, 107-131.

[19] Nalebuff, B. J., and J. E. Stiglitz, 1983, "Prizes and Incentives: Towards a General Theory of Compensation and Competition," Bell Journal of Economics, 14, 21-43.

[20] Negishi T. (1960). "Welfare economics and the existence of an equilibrium for a competitive economy". Metroeconomica 12, 92-97.

[21] Pfingsten A (1991). "Surplus sharing methods". Mathematical Social Sciences, 21, 287-301.

[22] Reynolds, (2000). "The One Member-One Vote rule in Cooperatives. Journal of Cooperatives, $15,7-62$.

[23] Roemer, J. and J. Silvestre, (1993), "The Proportional Solution for Economies with both Private and Public Ownership", Journal of Economic Theory, 59, 426-444.

[24] Sen, A. (1966), "Labour Allocation in a Cooperative Enterprise", Review of Economic Studies, $33,361-371$.

[25] Sobel, J. (2001). "On the Dynamics of Standards". Rand Journal of Economics, 32, 4, 606-623.

[26] Torregrosa-Montaner, R. (2017). "On the endogenous determination of the degree of meritocracy". Mimeo.

[27] Vanek, J. (1970). The General Theory of Labor-Managed Market Economies. Ithaca: Cornell University Press.

[28] Ward, B. (1958). "The Firm in Illyria: Market Syndicalism," American Economic Review, 48, 4, 566-89. 